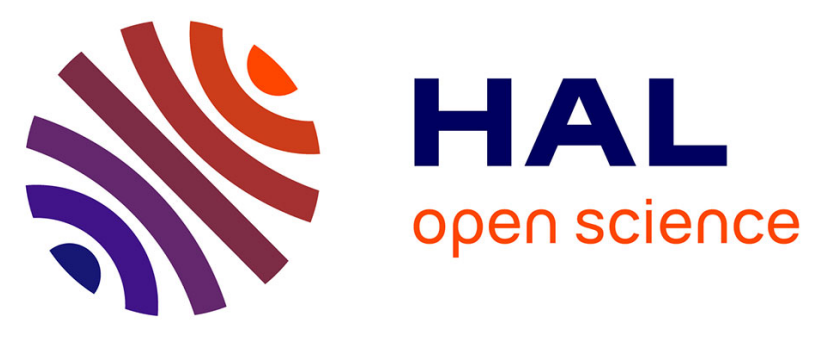

\title{
Extension of the measurement, assignment, and fit of the rotational spectrum of the two-top molecule methyl acetate
}

Ha Vinh Lam Nguyen, Isabelle Kleiner, Steven T Shipman, Yoshiaki Mae, Kazue Hirose, Shota Hatanaka, Kaori Kobayashi

\section{To cite this version:}

Ha Vinh Lam Nguyen, Isabelle Kleiner, Steven T Shipman, Yoshiaki Mae, Kazue Hirose, et al.. Extension of the measurement, assignment, and fit of the rotational spectrum of the two-top molecule methyl acetate. Journal of Molecular Spectroscopy, 2014, 299, pp.17-21. 10.1016/j.jms.2014.03.012 . hal-03183163

\section{HAL Id: hal-03183163 \\ https://hal.science/hal-03183163}

Submitted on 27 Mar 2021

HAL is a multi-disciplinary open access archive for the deposit and dissemination of scientific research documents, whether they are published or not. The documents may come from teaching and research institutions in France or abroad, or from public or private research centers.
L'archive ouverte pluridisciplinaire HAL, est destinée au dépôt et à la diffusion de documents scientifiques de niveau recherche, publiés ou non, émanant des établissements d'enseignement et de recherche français ou étrangers, des laboratoires publics ou privés. 


\title{
Extension of the Measurement, Assignment, and Fit of the Rotational Spectrum of the Two-Top Molecule Methyl Acetate
}

\author{
Ha Vinh Lam Nguyen ${ }^{a}$, Isabelle Kleiner ${ }^{a}$, Steven T. Shipman ${ }^{b}$, Yoshiaki Mae ${ }^{c}$, Kazue Hirose ${ }^{c}$, Shota \\ Hatanaka $^{c}$, and Kaori Kobayashic
}

a Laboratoire Interuniversitaire des Systèmes Atmosphériques (LISA), CNRS UMR 7583/IPSL et Universités Paris Est UPEC \& Paris Diderot UPD, Université de Paris Est, 61 avenue du Général de Gaulle, F-94010 Créteil cedex, France.

${ }^{\mathrm{b}}$ Division of Natural Sciences, New College of Florida, 5800 Bay Shore Road, Sarasota, FL 34243-2109, USA.

${ }^{\mathrm{c}}$ Department of Physics, Faculty of Science, Toyama University, 3190 Gofuku Toyama, Toyama, 930-8555, Japan.

Corresponding author: Dr. Ha Vinh Lam Nguyen

Phone: +331451765 53

Email: lam.nguyen@lisa.u-pec.fr

\begin{abstract}
New spectroscopic data were recorded for the two-top molecule methyl acetate using five spectrometers in four different labs: a room temperature chirped-pulse Fourier transform microwave (FTMW) spectrometer in the frequency range from 8.7 to $26.5 \mathrm{GHz}$, two molecular beam FTMW spectrometers (2 to $40 \mathrm{GHz}$ ), a free jet absorption Stark-modulated spectrometer (60 to $78 \mathrm{GHz}$ ), and a room temperature millimeter-wave spectrometer (44 to 68 $\mathrm{GHz}$ ). Approximately 800 new lines with $J$ up to 40 and $K$ up to 16 were assigned. In total, 1603 lines were fitted with 34 parameters using an internal rotation Hamiltonian in the Rho Axis Method (RAM) and the program BELGI-Cs-2tops to standard deviations close to the experimental uncertainties. More precise determinations of the top-top interaction and the $J, K$ dependent parameters were carried out.
\end{abstract}

Keywords: methyl acetate, chirped-pulse microwave spectroscopy, millimeter-wave spectroscopy, internal rotation, BELGI-Cs 


\section{Introduction}

Many organic compounds with internal rotation have been assigned and detected in interstellar space. The identification of most of those molecules was based on intense laboratory work followed by observations in the interstellar medium by means of microwave, millimeter-wave or submillimeter-wave telescopes. As an example, methanol has been found in Orion A [1]. Acetaldehyde was detected first in Sgr B2 [2] and then in the cold dust cloud TMC-1 and L134N [3]. The first detection of interstellar acetic acid was carried out by Mehringer et al. 1997 [4]. In 1975, Churchwell and Winnewisser reported on the detection of the $1_{10} \leftarrow 1_{11}$ transition of methyl formate $\mathrm{HCOOCH}_{3}$ in $\mathrm{Sgr} \mathrm{B} 2$, which arises as a doublet due to the internal rotation of the methyl group [5]. This molecule was also found in OrionKL in addition to methanol, dimethyl ether, acetonitrile, etc [6]. In 2007, Kobayashi et al. assigned seven lines observed using the Nobeyama $45 \mathrm{~m}$ radio telescope in the interstellar Orion KL cloud to the first torsional excited state $v_{t}=1$ of methyl formate [7]. Larger molecules like ethyl methyl ether (in the hot core region W51e2) [8, 9] and acetone [10] have also been detected.

Methyl acetate is an important molecule of astrophysical interest, since it has roughly the same size as molecules like ethyl methyl ether and acetone, which have already been detected in space. However, no report on the detection of methyl acetate in interstellar space could be found until last year, partly because the available spectroscopic data for this challenging molecule are not yet sufficient.

The challenge of analyzing the spectrum of methyl acetate is the internal rotation of two inequivalent methyl groups with one low $\left(102.413(20) \mathrm{cm}^{-1}\right)$ and one intermediate barrier (424.580(56) $\left.\mathrm{cm}^{-1}\right)$. Methyl acetate has been studied very early in 1980 by Sheridan and Bauder using Stark spectrometers in the frequency region from 8 to $40 \mathrm{GHz}$ [11]. The assignments were supported by microwave-microwave double resonance. About 100 lines with $J \leq 5$ and $K \leq 3$ were measured and fitted. In 2011, the investigation was considerably improved with 315 new lines recorded using the molecular beam Fourier transform microwave (MB-FTMW) spectrometers in Aachen, Germany and 519 lines recorded using the Free jet absorption Stark-modulated millimeter-wave (FJASMmm) spectrometer in Bologna, Italy [12]. A newly written program BELGI-Cs-2tops based on the Hamiltonian 
described by Ohashi et al. [13] was used to fit the complete data set to experimental precision using 27 molecular parameters.

During the course of the present work, it became obvious that using the parameters determined for the ground state $\mathrm{v}_{\mathrm{t}}=0$ of methyl acetate from [12], we were able to detect a large number of rotational transitions belonging to the five symmetry species $A, E_{1}, E_{2}, E_{3}$, and $\mathrm{E}_{4}$ (for nomenclature of the symmetry species see Refs. [12-13]) in the IRc2 region of the Orion KL nebula with the IRAM telescope [14]. These symmetry species arise from the interactions between the overall rotation and the internal rotations of both methyl tops as well as the interaction of the methyl tops with each other. In that paper, we extended the BELGICs-2tops code to be able to predict lines up to $J=30$. We also included intensity calculations for this two-top molecule.

However, some questions remain and have not yet been answered. First of all, in Ref. [12], the maximum $J$ and $K$ values for laboratory measurements were limited to $J=19$ and $K=7$. This also limits the uncertainties of the predicted lines. An extension of the measurements to higher $J$ and $K$ was not possible at that time due to the very low jet temperatures (approximately $1 \mathrm{~K}$ and $10 \mathrm{~K}$ for the MB-FTMW and the FJASMmm spectrometer, respectively). Therefore, many $J, K$ dependent parameters as well as the sextic centrifugal distortion constants could not be determined. Furthermore, some parameters, especially $V_{3}$ and $F$, are highly correlated and the $F$ values $\left(f_{1}, f_{2}\right.$, and $\left.f_{12}\right)$ had to be fixed to the values from the $a b$ initio geometry calculated at the MP2/6-311++G(d,p) level of theory. This level of theory is often used in the spectroscopic community to give reasonable starting rotational constants for spectrum assignment $[15,16]$. It is necessary to fit both $V_{3}$ and $F$ without too much correlation between these parameters. This can be achieved by including spectroscopic data of the torsional excited states. This method was successful in many one-rotor cases fitted with the BELGI-Cs-1top code [17,18] like acetaldehyde [19], acetic acid [20], acetamide [21], and methyl formate [22]. Furthermore, data on the torsional excited states for both tops are needed to test the two-top program (BELGI-Cs-2tops) which has not yet been used for that purpose.

For methyl acetate there are two torsional modes, which were calculated with the BELGI-Cs2tops code to be around $62.5 \mathrm{~cm}^{-1}$ for the $v_{27} \mathrm{C}_{-} \mathrm{CH}_{3}$ torsion and around $133.1 \mathrm{~cm}^{-1}$ for the $v_{26}$ $\mathrm{O}-\mathrm{CH}_{3}$ torsion. Recent ab initio calculations [23] located the $v_{26}$ mode at $137 \mathrm{~cm}^{-1}$ and the $v_{27}$ 
mode at $63 \mathrm{~cm}^{-1}$, which are in good agreement with our calculations. They also located three other small amplitude motions occurring at low frequencies, the $v_{25} \mathrm{C}-\mathrm{O}$ single-bond torsion mode at $237 \mathrm{~cm}^{-1}$, the $v_{17}$ in-plane COC bending mode at $334 \mathrm{~cm}^{-1}$, and the $v_{16}$ in-plane CCO bending mode at $484 \mathrm{~cm}^{-1}$. None of those bands were observed at high resolution so far due to the weakness of the bands. It is possible that a room temperature spectrum in the microwave range will contain rotational transitions within all those states because they will be populated. Fig. 1 shows a schematic of those lowest energy levels for methyl acetate up to $250 \mathrm{~cm}^{-1}$. It should be noted that the zero point energy of $99.94 \mathrm{~cm}^{-1}$ has been added to the vibrational mode frequencies in this Figure.

The first step to search for the $\mathrm{v}_{\mathrm{t}}=1$ transitions in a room temperature spectrum is to assign and fit all the higher $J$ and $K$ rotational transitions belonging to the ground torsional state. The goal of the present paper is to provide an improved set of parameters with which to predict the position of the $\mathrm{v}_{\mathrm{t}}=1$ lines to higher precision. This paper also extends the spectroscopic data available for astronomers by increasing the $J$ and $K$ values of the fit and the prediction to 40 and 16 , respectively.

\section{Experimental setup}

\subsection{Millimeter-wave measurements}

The conventional source modulation spectrometer was used to record the 44 to $68 \mathrm{GHz}$ region at the University of Toyama, Japan. The detail of this spectrometer has been already described elsewhere [24] and will be only explained very briefly here. The old synthesized sweeper (HP 83642A) in Ref. [24] was replaced by a new signal generator (Agilent Technologies E8257D). The microwave radiation from this signal generator was used in combination with multipliers and detected with a liquid He-cooled InSb detector. The source was modulated at $50 \mathrm{kHz}$ and demodulated at $100 \mathrm{kHz}$ with a lock-in amplifier. The sample pressure in the waveguide cell was maintained at $30-50$ mTorr.

\subsection{Chirped-pulsed measurements}

The room temperature chirped-pulse Fourier transform microwave spectrometer at New College of Florida, USA, has been previously described in detail [25], but a brief description follows. Data were collected in three frequency regions: $8.7-13.5,13.5-18.3$, and $18-26.5$ GHz. In each region, a chirped pulse is produced by an arbitrary waveform generator (Tektronix AWG7101). This pulse is filtered, frequency shifted to the region of interest by 
mixing with a local oscillator source, filtered again, and then amplified before being sent into a 10-meter long WRD-750 waveguide. The waveguide contained methyl acetate at a pressure of roughly 6 mTorr. The signal resulting from interactions of the methyl acetate sample with the chirped pulse was amplified with a high-gain, low-noise amplifier, frequency shifted to either $0.1-4.9 \mathrm{GHz}(8.7-13.5$ or $13.5-18.3 \mathrm{GHz}$ circuits $)$ or $0.7-9.2 \mathrm{GHz}(18-26.5 \mathrm{GHz}$ circuits) with a local oscillator source, and finally amplified and filtered again before digitization with a high-speed oscilloscope (Tektronix TDS6154C). The arbitrary waveform generator, oscilloscope, and all local oscillators were locked to a $10 \mathrm{MHz}$ external reference (Stanford Research Systems FS725) to ensure phase stability of the signal from cycle to cycle.

The exact filters, amplifiers, and local oscillators used in the spectrometer vary based on frequency region, but the overall operation of the spectrometer is the same in each. In each frequency region, the molecular free induction decay was collected for 4 microseconds and averaged for 2.5 million cycles before being Fourier transformed to yield the frequencydomain spectrum. The 4 microsecond collection time is the primary source of line broadening in measurements with this instrument and leads to peaks in the frequency domain with line widths of roughly $600 \mathrm{kHz}$.

\section{Spectrum assignment and fits}

The spectroscopic constants from the previous fit published in Ref. [12] were used to predict the transition frequencies in the region from 44 to $68 \mathrm{GHz}$ which can be recorded with the spectrometer in Toyama. It should be noted that no measurements were carried out in the frequency range 54.4 to $57.4 \mathrm{GHz}$. All five torsional components of many transitions up to $J$ $=15$ and $K=7$ could be directly found and fitted without any additional parameter to an excellent root-mean-square deviation of $57.1 \mathrm{kHz}$ for 377 transitions, almost within our experimental uncertainty of $50 \mathrm{kHz}$. These transitions are important, since they are in the missing region between the microwave region of the spectrometers in Aachen and the millimeter-wave region of the spectrometer in Bologna and therefore could not be previously measured. A small part of the Toyama spectrum is given in Fig. 2.

As a next step, the program BELGI-Cs-2tops was slightly modified to a version which can predict and calculate transitions up to $J=40$ (instead of the previous versions which went up to $J=20$ [12] or $J=30$ [14]). Initially, we only predicted transitions up to $J=25$. The 
intensity calculations were taken from two BELGI-Cs-1top fits, where the internal rotation of one or the other methyl groups is neglected. The assignment was started with the A doublets of the $R$-branch $(J+1)\left(K_{\mathrm{a}}-1\right) \pm \leftarrow J K_{\mathrm{a}} \pm(J \geq 15)$. We searched the room temperature CPFTMW spectrum around the predicted frequency regions for doublets with approximately equal intensity which were predicted to be strong using the BELGI-Cs-1top fits. This is important since the room temperature spectrum is, as shown in Fig. 3, very crowded. It is thus sometimes very difficult to decide which among several neighboring lines is the correct transition. It is no longer satisfactory to only use the calculated frequencies to assign the spectrum for $J \geq 15$, since the prediction of the previous fit from Ref. [12] is only accurate for transitions with lower $J$ and $K$. After the A species transitions were found and fitted, some $\mathrm{E}_{2}$ species were added step by step, since the $\mathrm{A}-\mathrm{E}_{2}$ torsional splittings (from the larger torsional barrier) are smaller and therefore easier to assign. For the nomenclature of the symmetry species see Ref. [12]. The parameters were improved after adding these transitions. Afterwards, the $\mathrm{E}_{1}$ species (arising from the smaller torsional barrier) could also be assigned and finally, we included the $\mathrm{E}_{3}$ and $\mathrm{E}_{4}$ transitions in the fit.

As mentioned above, the A-E $E_{1}$ splittings of many transitions are very large due to the low barrier to internal rotation of the acetyl methyl group. Therefore, many $E_{1}, E_{3}$, and $E_{4}$ torsional states are outside the measured frequency range. For such transitions, only the A and $\mathrm{E}_{2}$ species could be given.

As a next step, we extended the prediction using the new parameters to $J=40$, repeated the process, and again succeeded by trial and error to fit the whole data set containing 377 new lines from the millimeter-wave instrument in Toyama and 386 new lines from the chirpedpulse instrument in Florida. Due to the improvement of the parameters, we were also able to add new transitions from the initial Aachen and Bologna measurements. The final fit with a total of 1603 transitions reproduces the measured transitions using 34 parameters to a unitless standard deviation of 0.97. Compared to Ref. [12], seven additional parameters were used. All of them are $J, K$ dependent parameters.

An overview of the data sets with their respective standard deviations is shown in Table 1 and the molecular parameters are given in Table 2. A list of all fitted transitions is available as supplementary material (Table S-1 and S-2). 


\section{Discussion}

The present work has doubled the maximum $J$ and $K$ values as well as the number of transitions, compared to Ref. [12]. Those transitions with higher $J$ and $K$ have significantly improved the predictive power of the fit. Many predicted frequencies for higher $J$ transitions using the old parameters from the lower $J$ (up to $J=19$ ) fit in Ref. [12] are already off by up to $1 \mathrm{MHz}$ at $J \leq 25$ (see Table 3 ). Since the spectra at room temperature are very dense, it was necessary to check all lines carefully.

It should be mentioned that the $J, K$ distribution of the new lines is not uniform, since the new lines are mostly ${ }^{\mathrm{r}} \mathrm{P}$ or ${ }^{\mathrm{P}} \mathrm{R}$ lines (in symmetric top notation), which must satisfy $v=\mathrm{E}$ ' $-\mathrm{E}^{\prime \prime} \approx 0$ in order to fall in the microwave or millimeter-wave region. This requires

$\left[B(J+1)(J+2)+(A-B) K^{2}\right]-\left[B J(J+1)+(A-B)(K+1)^{2}\right] \approx 0$,

which then requires that $K$ and $J$ have integer values such that $K \approx J /(A / B-1)-1 / 2$. Because $K$ is a fixed function of $J$, there is significant correlation between $D_{\mathrm{J}}, D_{\mathrm{JK}}$, and $D_{\mathrm{K}}$, which can essentially all be converted to contributions to $D_{\mathrm{J}}$. This may explain why the centrifugal distortion parameters determined by the present work show some differences when compared to the previous set of values.

In total, 767 new lines but only 7 new parameters compared to Ref. [12] were added. These seven new parameters can be thought of as the $J$ and $K$ dependence of various lower order terms in the torsion-rotation Hamiltonian as shown in Table 2. Nevertheless, BELGI-Cs-2tops works very well in the observed $J$ and $K$ range and fits almost all lines to experimental measurement accuracy (see Table 1) with a very acceptable ratio of 47.1 lines/parameter.

The present fit decorrelates $V_{31}, V_{32}, V_{12 \mathrm{~s}}$, and $V_{12 \mathrm{c}}$, but not $f_{1}, f_{2}$, and $f_{12}$ which were kept fixed as in [12]. To break these correlations, information from higher torsional states is necessary. Our plans for future work are thus to attack the first excited state of each of the two torsional modes in this molecule using the room temperature microwave spectra, even if it is not the optimal solution, since many energy levels are occupied and many transitions can be observed in the spectrum. The extension of the ground state spectrum of methyl acetate was a necessary first step since many ground state transitions must be subtracted from these spectra. 


\section{Acknowledgement}

We thank the PROCOPE project of DAAD for traveling money, Japan Society Promotion Science, Invitation Fellowship Programs for Research in Japan (ID no. S120124), and the ANR-08-BLAN-0054 TopModel project for funds. S.T.S. acknowledges support from the National Science Foundation Division of Chemistry under Grant No. 1111101 (co-funded by MPS/CHE and the Division of Astronomical Sciences). I.K. and H.V.L.N. would like to thank Dr. Manceron and Dr. Kwabia for their efforts in trying to record the far-infrared spectra at the synchrotron Soleil, St. Aubin, France.

\section{References}

[1] F.J. Lovas, D.R. Johnson, D. Buhl, L.E. Snyder, Astrophys. J. 209 (1976) 770.

[2] M.B. Bell, H.E. Matthews, P.A. Feldman, Astron. Astrophys. 127 (1983) 420.

[3] H.E. Matthews, P. Friberg, W.M. Irvine, Astron. Astrophys. 290 (1985) 609.

[4] D.M. Mehringer, L.E. Snyder, Y. Miao, F.J. Lovas, Astrophys. J. 480 (1997) 71.

[5] E. Churchwell and G. Winnewisser, Astron. Astrophys. 45 (1975) 229.

[6] C.W. Lee, S.H. Cho, S.M. Lee, Astrophys. J. 551 (2001) 333.

[7] K. Kobayashi, K. Ogata, S. Tsunekawa, S. Takano, Astrophys. J. 657 (2007) L17.

[8] G.W. Fuchs, U. Fuchs, T.F. Giesen, F. Wyrowski, Astron. Astrophys. 444 (2) (2005) 521.

[9] K. Kobayashi, T. Matsui , N. Mori , S. Tsunekawa , N. Ohashi, J. Mol. Spectrosc. 251 (2008) 301.

[10] F. Combes, M. Gerin, A. Wootten, G. Wlodarczak, F. Clausset, P.J. Encrenaz, Astron. Astrophys. 180 (1987) 13.

[11] J. Sheridan, W. Bossert, A. Bauder, J. Mol. Spectrosc. 80 (1980) 1.

[12] M. Tudorie, I. Kleiner, J.T. Hougen, S. Melandri, L.W. Sutikdja, W. Stahl, J. Mol. Spectrosc. 269 (2011) 211.

[13] N. Ohashi, J.T. Hougen, R.D. Suenram, F.J. Lovas, Y. Kawashima, M. Fujitake, J. Pyka, J. Mol. Spectrosc. 227 (2004) 28.

[14] B. Tercero, I. Kleiner, J. Cernicharo, H.V.L. Nguyen, A. López, G.M. Munoz Caro, ApJ. 770 (2013) L13.

[15] H.V.L. Nguyen, W. Stahl, J. Mol. Spectrosc. 264 (2010) 120.

[16] H.V.L. Nguyen, R. Kannengiesser, W. Stahl, Phys. Chem. Chem. Phys. 14, 11753 (2012).

[17] J.T. Hougen, I. Kleiner, M. Godefroid, J. Mol. Spectrosc. 163 (1994) 559. 
[18] I. Kleiner, J. Mol. Spectrosc. 260 (2010) 1.

[19] I. Kleiner, J.T. Hougen, J.-U. Grabow, S.P. Belov, M.Y. Tretyakov, J. Cosleou , J. Mol. Spectrosc. 179 (1996) 41.

[20] V.V. Ilyushin, E.A. Alekseev, S.F. Dyubko, I. Kleiner, J. Mol. Spectrosc. 220 (2003) 170 .

[21] V.V. Ilyushin, E.A. Alekseev, S.F. Dyubko, I. Kleiner, J.T. Hougen, J. Mol. Spectrosc. 227 (2004) 115.

[22] M. Carvajal, F. Willaert, J. Demaison, I. Kleiner, J. Mol. Spectrosc. 246 (2007) 158.

[23] M.L. Senent, R. Domínguez-Gómez, M. Carvajal, I. Kleiner, J. Chem. Phys. 138 (2013) 044319.

[24] Y. Fukuyama, H. Odashima, K. Takagi, S. Tsunekawa, Astrophys. J. Suppl. 104 (1996) 329.

[25] B. Reinhold, I.A. Finneran, S.T. Shipman, J. Mol. Spectrosc. 270 (2011) 89. 


\section{Figure}

Figure 1: The lowest energy levels for methyl acetate at $J=K=0$ with their symmetry species using the notations in Ref. [12] and [13]. For the ground torsional state $\left(v_{t}=0\right)$ the zero point energy is located at $99.94 \mathrm{~cm}^{-1}$ and the location of its five symmetry species is shown on the right. The first excited torsional states $\mathrm{v}_{27}=1$ and $\mathrm{v}_{26}=1$ are extrapolated using our parameters to be at 162.49 and $233.02 \mathrm{~cm}^{-1}$, respectively. The second torsional excited state $\mathrm{v}_{27}=2$ is also presented in this figure. They agree with those values by $a b$ initio calculations [23]. We note that none of the torsional bands or rotational transitions belonging to these torsional excited states have been measured so far.

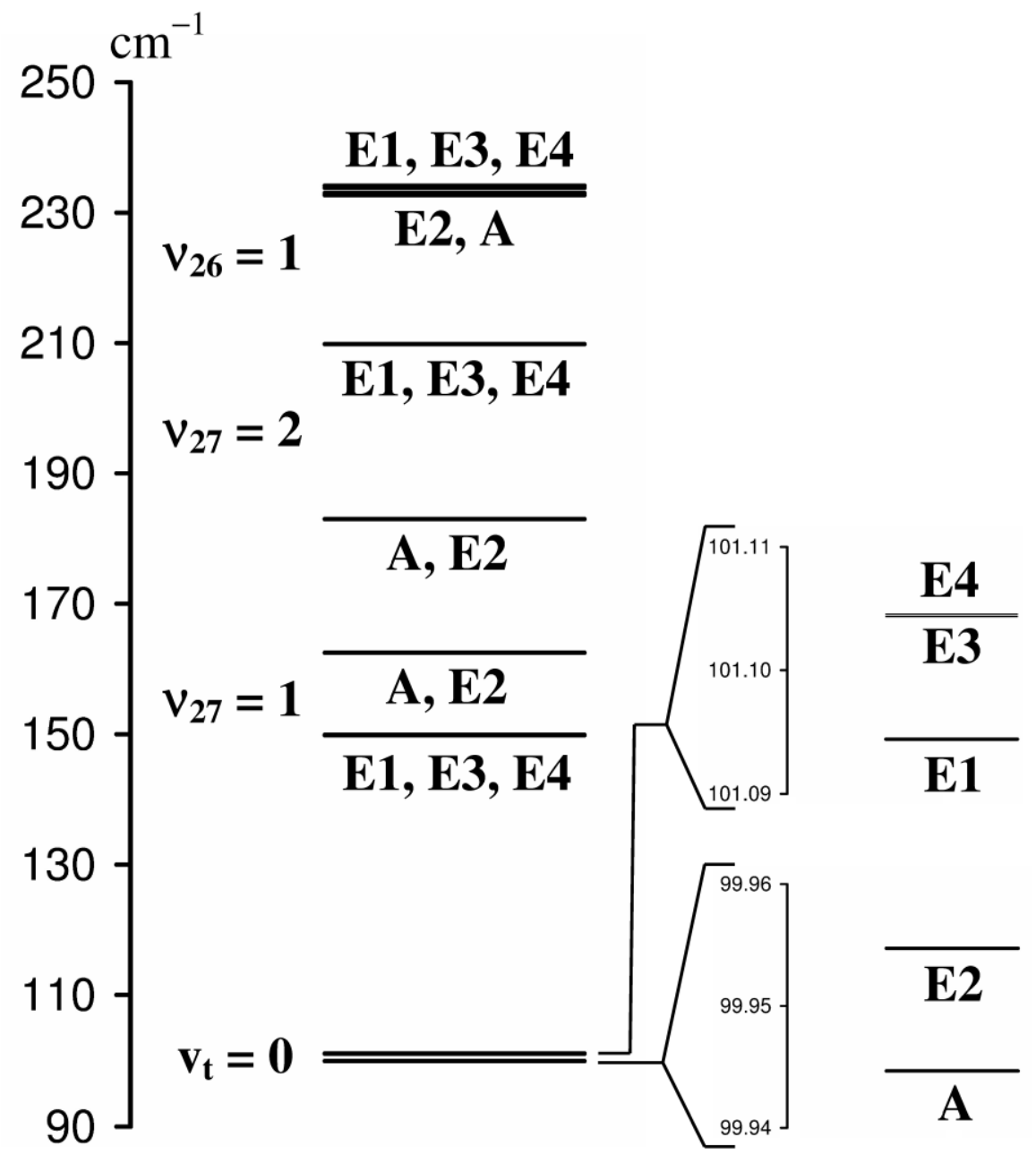


Figure 2: A stick diagram from the room temperature millimeter-wave spectrum of methyl acetate in the frequency range of $300 \mathrm{MHz}$ from $52200 \mathrm{MHz}$ to $52500 \mathrm{MHz}$ recorded in Toyama, Japan (lower trace). The calculated spectrum is in the upper trace. The assigned transitions $J^{\prime}{ }^{\prime} a^{\prime} \leftarrow J^{\prime \prime}{ }_{K a}$ " are marked. A, $\mathrm{E}_{1}, \mathrm{E}_{2}, \mathrm{E}_{3}$, and $\mathrm{E}_{4}$ are the torsional species due to internal rotations of the two methyl groups (see text). For the A species the "parity" quantum number + and - have been added. For the E species, the sign of $K_{\mathrm{a}}$ is indicated following the notation of [17] and references therein. The intensities are given in arbitrary units. The measurement accuracy is $50 \mathrm{kHz}$.

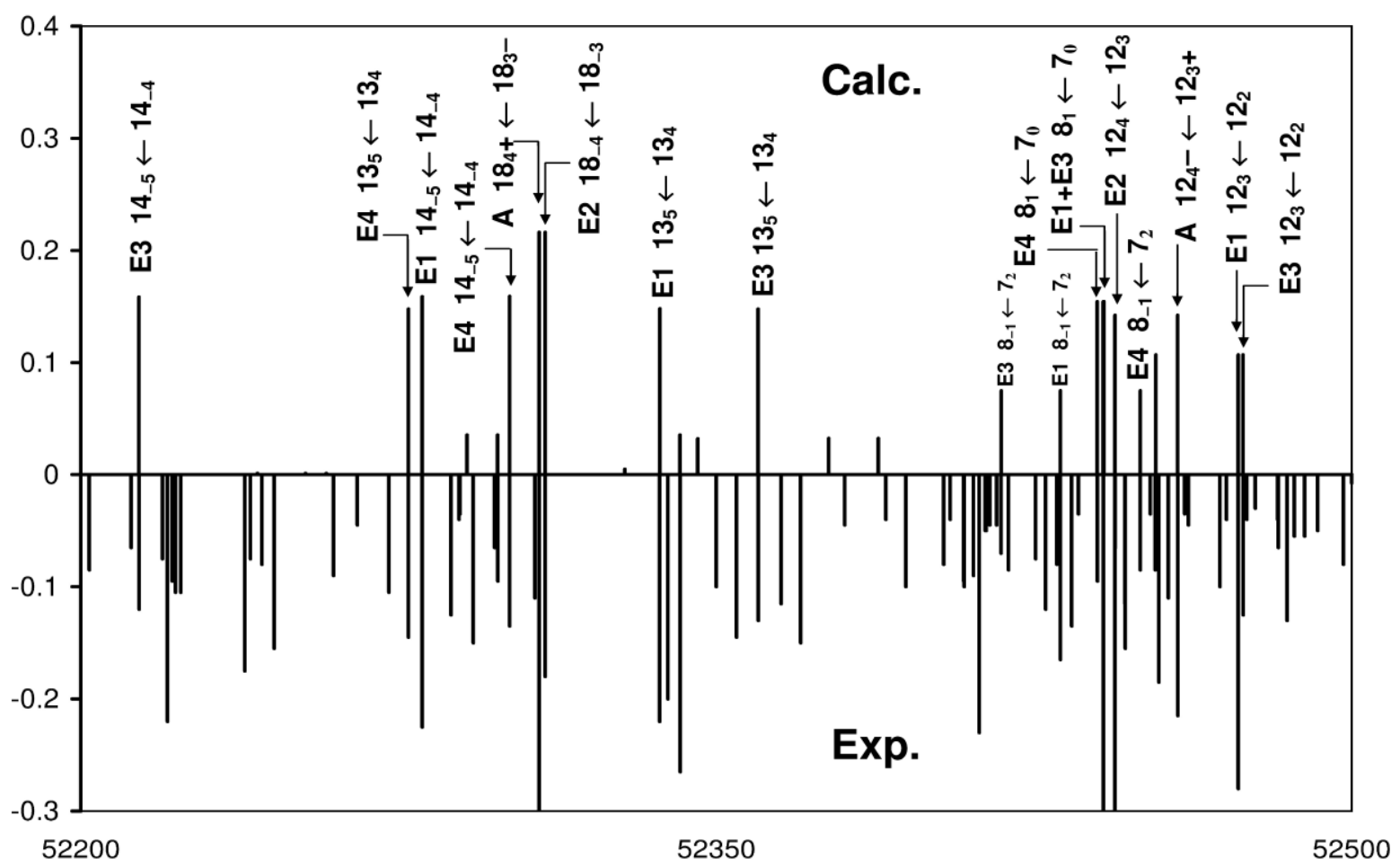


Figure 3: A scan from the room temperature CP-FTMW spectrum of methyl acetate in the frequency range from $13810 \mathrm{MHz}$ to $13850 \mathrm{MHz}$ recorded in Florida, USA. A and $\mathrm{E}_{2}$ species refer to the smaller splittings due to internal rotation of the methoxy methyl group. The intensities are given in arbitrary units. The line width is about $600 \mathrm{kHz}$, depending on the respective transitions, since some transitions overlap. The measurement accuracy is $80 \mathrm{kHz}$. The assigned transitions follow the same notation as in Fig. 2.

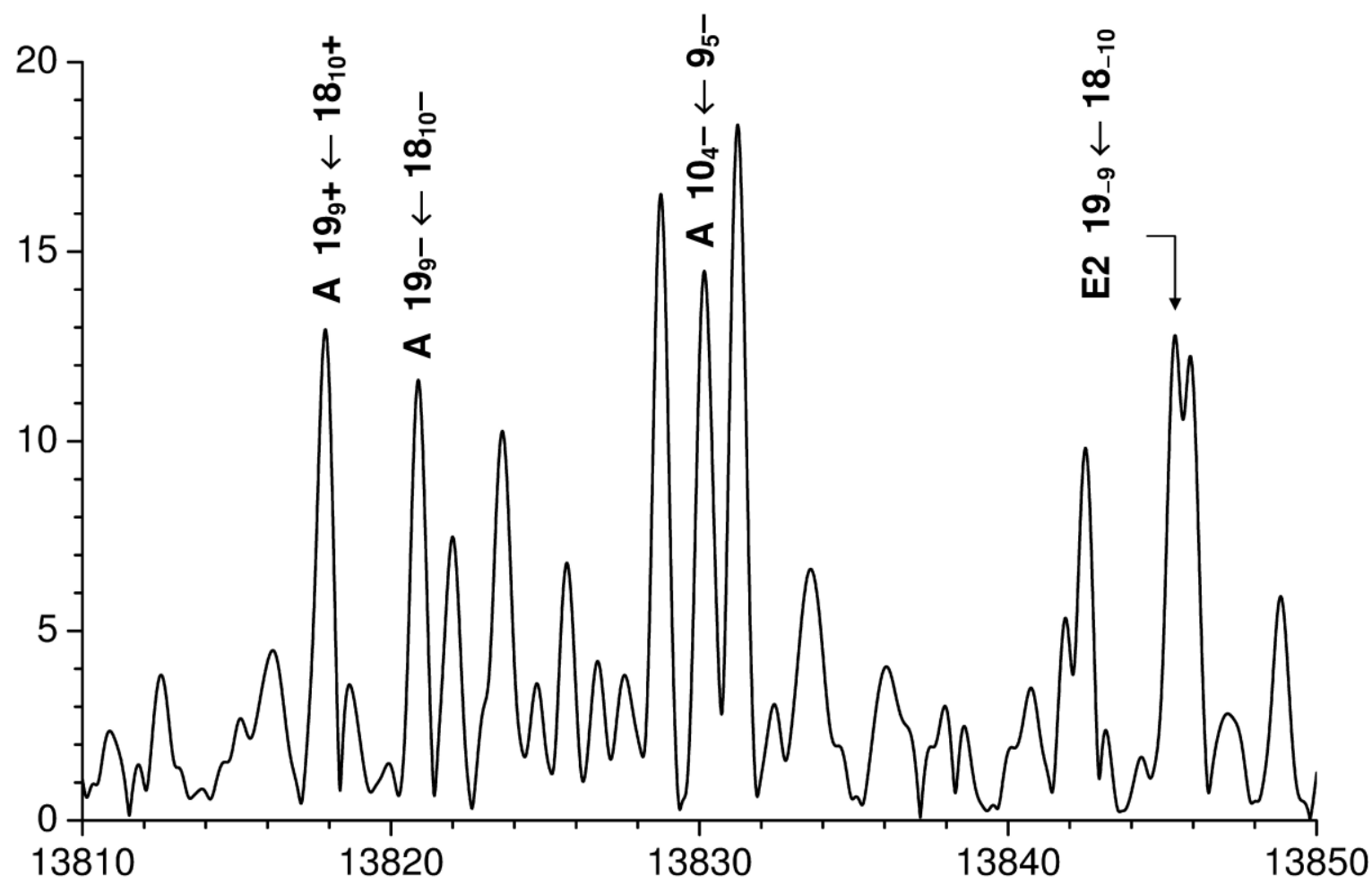




\section{Table}

Table 1: Overview of the data set coverage ${ }^{\mathrm{a}}$ and fit quality.

\begin{tabular}{lllllllll}
\hline & \multicolumn{3}{c}{ MB-FTMW lines $^{\mathrm{b}}$} & \multicolumn{5}{c}{ FJASMmm-wave lines $^{\mathrm{c}}$} \\
& \multicolumn{3}{c}{$2.0-40.0 \mathrm{GHz}$} & \multicolumn{4}{c}{$59.8-77.3 \mathrm{GHz}$} \\
\hline$\Gamma^{\mathrm{d}}$ & $\#^{\mathrm{e}}$ & $\mathrm{rms}^{\mathrm{f}}$ & $J_{\max }{ }^{\mathrm{g}}$ & $K_{\max }{ }^{\mathrm{g}}$ & $\#^{\mathrm{e}}$ & $\mathrm{rms}^{\mathrm{f}}$ & $J_{\max }{ }^{\mathrm{g}}$ & $K_{\max }{ }^{\mathrm{g}}$ \\
$\mathrm{A}$ & 63 & 2.7 & 9 & 3 & 96 & 30.0 & 17 & 7 \\
$\mathrm{E}_{1}$ & 64 & 3.9 & 9 & $+3,-3$ & 123 & 24.7 & 19 & $+7,-6$ \\
$\mathrm{E}_{2}$ & 65 & 3.1 & 9 & $+3,-3$ & 107 & 30.5 & 17 & $+6,-6$ \\
$\mathrm{E}_{3}$ & 61 & 2.8 & 9 & $+3,-3$ & 98 & 34.7 & 18 & $+6,-6$ \\
$\mathrm{E}_{4}$ & 63 & 4.0 & 9 & $+3,-3$ & 98 & 31.8 & 19 & $+7,-6$ \\
\hline
\end{tabular}

\begin{tabular}{lllllllll}
\hline \multicolumn{4}{c}{ RT CP-FTMW lines $^{\mathrm{h}}$} & \multicolumn{4}{c}{ RT Mm-wave lines $^{\mathrm{i}}$} \\
\multicolumn{4}{c}{$8.7-26.5 \mathrm{GHz}$} & \multicolumn{3}{c}{$44.0-68.0 \mathrm{GHz}$} \\
\hline$\Gamma^{\mathrm{d}}$ & $\#^{\mathrm{e}}$ & $\mathrm{rms}^{\mathrm{f}}$ & $J_{\max }{ }^{\mathrm{g}}$ & $K_{\max }{ }^{\mathrm{g}}$ & $\#^{\mathrm{e}}$ & $\mathrm{rms}^{\mathrm{f}}$ & $J_{\max } \mathrm{g}^{\mathrm{g}}$ & $K_{\max }{ }^{\mathrm{g}}$ \\
$\mathrm{A}$ & 76 & 70.6 & 30 & 16 & 63 & 51.1 & 30 & 13 \\
$\mathrm{E}_{1}$ & 88 & 88.9 & 40 & $+12,-13$ & 86 & 57.6 & 39 & $+12,-11$ \\
$\mathrm{E}_{2}$ & 99 & 88.4 & 33 & $+15,-14$ & 90 & 61.5 & 37 & $+12,-12$ \\
$\mathrm{E}_{3}$ & 65 & 95.7 & 33 & $+10,-13$ & 68 & 53.9 & 38 & $+12,-8$ \\
$\mathrm{E}_{4}$ & 58 & 94.0 & 28 & $+13,-13$ & 70 & 58.7 & 39 & $+13,-15$ \\
\hline \hline
\end{tabular}

${ }^{a}$ This includes two lines from Ref. [11] at 25733.190 and $25786.250 \mathrm{MHz}$, which were fitted in Ref. [12].

${ }^{\mathrm{b}}$ These 316 Aachen measurements (Molecular-Beam Fourier Transform Microwave) fit to their measurement error (4 kHz); one line was added compared to Ref. [12].

${ }^{\mathrm{c}}$ These 522 Bologna measurements (Free Jet Absorption Millimeter) fit to their measurement error (40 kHz); three lines were added compared to Ref. [12].

${ }^{\mathrm{d}}$ Symmetry species of the lines in each row.

${ }^{\mathrm{e}}$ Number of lines in each data group.

${ }^{\mathrm{f}}$ Root-mean-square deviation of each data group in $\mathrm{kHz}$.

${ }^{\mathrm{g}}$ Largest $J$ and $K_{a}$ values in each data group (for E species the largest positive and negative $K_{a}$ values are both given). Smallest $J$ and $K_{a}$ values are all 0, except for FJASMmm-wave lines which have $J_{\min }=3$.

${ }^{\mathrm{h}}$ These 386 Florida measurements (Room Temperature Chirped-pulsed Fourier Transform) fit to almost their measurement error $(80 \mathrm{kHz})$.

${ }^{\mathrm{i}}$ These 377 Toyama measurements (Room Temperature Millimeter) fit to their measurement error $(50 \mathrm{kHz})$. 
Table 2: Molecular parameters of methyl acetate obtained with the program BELGI-Cs-2tops (all values are given in $\mathrm{cm}^{-1}$ ).

\begin{tabular}{|c|c|c|c|c|}
\hline Operator $^{\mathrm{a}}$ & $\mathrm{b}$ & c & From Ref. [12] ${ }^{\mathrm{d}}$ & Present fit \\
\hline$J_{z}^{2}$ & $A$ & $O A$ & $0.3818404(90)$ & $0.383358(48)$ \\
\hline$J_{x}^{2}$ & $B$ & $B$ & $0.1401459(17)$ & 0.146944 (19) \\
\hline$J_{y}^{2}$ & C & $C$ & $0.1025080(15)$ & 0.109312 (19) \\
\hline$-\mathbf{J}^{4}$ & $\Delta_{J} \cdot 10^{7}$ & $D J$ & $0.23115(4)$ & $0.2840(28)$ \\
\hline$-\mathbf{J}^{2} J_{z}^{2}$ & $\Delta_{J K} \cdot 10^{7}$ & $D J K$ & $-0.321(21)$ & $15.061(87)$ \\
\hline$-J_{z}^{4}$ & $\Delta_{K} \cdot 10^{6}$ & $D K$ & $-0.602(68)$ & $-0.189(42)$ \\
\hline$-2 \mathbf{J}^{2}\left(J_{x}^{2}-J_{y}^{2}\right)$ & $\delta_{J} \cdot 10^{8}$ & ODELN & $0.5973(1)$ & $1.202(13)$ \\
\hline$-2 J_{x}^{2}\left(J_{x}^{2}-J_{y}^{2}\right)$ & $\delta_{K} \cdot 10^{9}$ & $O D E L K$ & Not used & $-0.94(90)$ \\
\hline$p_{1}^{2}$ & $f_{1}$ & $F 1$ & $5.554669^{e}$ & $5.554669^{\mathrm{e}}$ \\
\hline$p_{2}^{2}$ & $f_{2}$ & $F 2$ & $5.523464^{\mathrm{e}}$ & $5.523464^{\mathrm{e}}$ \\
\hline$p_{1} p_{2}$ & $f_{12}$ & $F 12$ & $0.664^{\mathrm{e}}$ & $0.664^{\mathrm{e}}$ \\
\hline$(1 / 2)\left(1-\cos 3 \alpha_{1}\right)$ & $V_{3,1}$ & $V 31$ & $101.740(30)$ & $102.413(20)$ \\
\hline$\left(1-\cos 3 \alpha_{1}\right) J_{z}^{2}$ & $V_{3,1 K}$ & $V 31 K$ & $0.0021793(95)$ & $0.001962(20)$ \\
\hline$(1 / 2)\left(1-\cos 3 \alpha_{2}\right)$ & $V_{3,2}$ & $V 32$ & $422.148(55)$ & $424.581(56)$ \\
\hline$\left(1-\cos 3 \alpha_{1}\right)\left(1-\cos 3 \alpha_{2}\right)$ & $V_{12 c}$ & $V 12 C$ & $-3.526(26)$ & $-4.236(37)$ \\
\hline $\sin 3 \alpha_{1} \sin 3 \alpha_{2}$ & $V_{12 s}$ & $V 12 S$ & $34.24(28)$ & $39.14(10)$ \\
\hline$J_{z} p_{1}$ & $q_{1}$ & $Q 1$ & $-0.68129(12)$ & $-0.68242(10)$ \\
\hline$J_{z} p_{2}$ & $q_{2}$ & $Q 2$ & $-0.70434(10)$ & $-0.71463(28)$ \\
\hline$J_{z}^{3} p_{2}$ & $q_{2 K} \cdot 10^{4}$ & $Q 2 K$ & $-0.148(11)$ & $-0.4210(62)$ \\
\hline$J_{x} p_{1}$ & $r_{1}$ & $R 1$ & $-0.124446(42)$ & $-0.124561(31)$ \\
\hline $\mathbf{J}^{2} J_{x} p_{1}$ & $r_{1 J} \cdot 10^{6}$ & $R 1 J$ & $-0.2155(64)$ & $-0.6923(32)$ \\
\hline$J_{x} p_{2}$ & $r_{2}$ & $R 2$ & $-0.092967(28)$ & $-0.093989(38)$ \\
\hline$(1 / 2)\left(J_{z}^{2} J_{x}+J_{x} J_{z}^{2}\right) p_{2}$ & $r_{2 K} \cdot 10^{4}$ & $R 2 K$ & $-0.2098(35)$ & $-0.2696(29)$ \\
\hline$J_{z} p_{1} p_{2}\left(p_{1}+p_{2}\right)$ & $q_{12} \cdot 10^{3}$ & $Q 12 P$ & $-0.212(11)$ & $-0.313(19)$ \\
\hline$J_{x} p_{1} p_{2}\left(p_{1}+p_{2}\right)$ & $r_{12 p} \cdot 10^{3}$ & $R 12 P$ & $0.1643(63)$ & 0.1867 (49) \\
\hline$J_{x}^{2} p_{1}^{2}$ & $B_{1} \cdot 10^{4}$ & $B 1$ & $0.29552(79)$ & $0.14113(56)$ \\
\hline$J_{x}^{2} p_{2}^{2}$ & $B_{2} \cdot 10^{4}$ & $B 2$ & $0.1878(29)$ & $-4.876(13)$ \\
\hline$J_{x}^{2} p_{1} p_{2}$ & $B_{12} \cdot 10^{4}$ & $B 12$ & $-0.4979(61)$ & $-0.1930(26)$ \\
\hline$J_{y}^{2} p_{1}^{2}$ & $C_{1} \cdot 10^{5}$ & $C C 1$ & $0.7308(31)$ & $-0.8606(19)$ \\
\hline$J_{y}^{2} p_{2}^{2}$ & $C_{2} \cdot 10^{4}$ & $C C 2$ & $0.1760(26)$ & $-4.917(14)$ \\
\hline$J_{y}^{2} p_{1} p_{2}$ & $C_{12} \cdot 10^{4}$ & $C C 12$ & $-0.2424(40)$ & $0.0190(15)$ \\
\hline$\left(1-\cos 3 \alpha_{2}\right) J_{z}^{2}$ & $V_{3,2 K}$ & $V 32 K$ & Not used & $0.017584(16)$ \\
\hline $\mathbf{J}^{2} J_{x} p_{2}$ & $r_{2 J} \cdot 10^{5}$ & $R 2 J$ & Not used & 0.6357 (59) \\
\hline $\mathbf{J}^{2} p_{2}$ & $q_{2 J} \cdot 10^{4}$ & $Q 2 J$ & Not used & $0.56048(19)$ \\
\hline$J_{z}^{3} p_{1}$ & $q_{1 K} \cdot 10^{5}$ & $Q 1 K$ & Not used & $0.455(22)$ \\
\hline$(1 / 2)\left(J_{z}^{2} J_{x}+J_{x} J_{z}^{2}\right) p_{1}$ & $r_{1 K} \cdot 10^{6}$ & $R 1 K$ & Not used & $-0.637(65)$ \\
\hline $\mathbf{J}^{2}\left(1-\cos 3 \alpha_{2}\right)$ & $V_{3,2 J}$ & $V 32 J$ & Not used & $-0.022807(61)$ \\
\hline
\end{tabular}

${ }^{a}$ Operator which the parameter multiplies in the program. $\alpha_{1}$ and $\alpha_{2}$ correspond to the $\mathrm{CH}_{3}-\mathrm{C}$ and the $-\mathrm{O}-\mathrm{CH}_{3}$ methyl group, respectively.

${ }^{\mathrm{b}}$ Notation of Eq. (6) and Table 3 of Ref. [13], except for $A, B$, and $C$, where the prime was removed.

${ }^{\mathrm{c}}$ Notation used in the program input and output.

${ }^{\mathrm{d}}$ Value of the parameter obtained from the final least-squares fit, with one standard uncertainty given in parentheses. 
${ }^{\mathrm{e}}$ These parameters were held fixed in the fit.

Table 3: The predicted frequencies of two A-doublet $R$ branches in the room temperature CPFTMW spectrum (with an experimental uncertainty of $80 \mathrm{kHz}$ ) using the old parameters from Ref. [12] ( $\left.v_{\text {Calc. }}{ }^{a}\right)$ and using the parameters from this present paper $\left(v_{\text {Calc. }}{ }^{b}\right)$. $v_{\text {Obs. }}$ is the observed frequency.

\begin{tabular}{|c|c|c|c|c|c|c|c|c|c|c|}
\hline \multicolumn{4}{|c|}{ Upper level } & \multicolumn{4}{|c|}{ Lower level } & \multirow{2}{*}{$\begin{array}{l}\text { Vobs. } \\
\text { MHz }\end{array}$} & \multirow{2}{*}{$\begin{array}{c}v_{\text {Obs. }}-v_{\text {Calc. }}{ }^{\mathrm{a}} \\
\mathrm{MHz}\end{array}$} & \multirow{2}{*}{$\begin{array}{c}v_{\text {Obs. }}-v_{\text {Calc. }}{ }^{b} \\
\mathrm{MHz}\end{array}$} \\
\hline$J$ & $K_{\mathrm{a}}$ & $K_{\mathrm{c}}$ & $P$ & $J$ & $K_{\mathrm{a}}$ & $K_{\mathrm{c}}$ & $P$ & & & \\
\hline 13 & 6 & 7 & + & 12 & 7 & 6 & + & 9381.6382 & -0.1072 & 0.066 \\
\hline 15 & 7 & 8 & - & 14 & 8 & 7 & - & 10809.8247 & 0.055 & -0.010 \\
\hline 17 & 8 & 9 & + & 16 & 9 & 8 & + & 12293.9311 & 0.4394 & -0.037 \\
\hline 19 & 9 & 10 & - & 18 & 10 & 9 & - & 13820.8831 & 1.3853 & -0.012 \\
\hline 21 & 10 & 11 & + & 20 & 11 & 10 & + & 15387.3294 & 3.6137 & -0.072 \\
\hline 23 & 11 & 12 & - & 22 & 12 & 11 & - & 16993.6020 & Not predicted & 0.125 \\
\hline 13 & 6 & 8 & - & 12 & 7 & 5 & - & 9289.1807 & 0.0037 & -0.042 \\
\hline 15 & 7 & 9 & + & 14 & 8 & 6 & + & 10779.8768 & -0.0654 & 0.112 \\
\hline 17 & 8 & 10 & - & 16 & 9 & 7 & + & 12284.4085 & 0.4135 & -0.010 \\
\hline 19 & 9 & 11 & + & 18 & 10 & 8 & + & 13817.8646 & 1.4262 & -0.052 \\
\hline 21 & 10 & 12 & - & 20 & 11 & 9 & + & 15386.4373 & 3.5899 & -0.047 \\
\hline 23 & 11 & 13 & + & 22 & 12 & 10 & + & 16993.0920 & Not predicted & -0.106 \\
\hline
\end{tabular}

